KEK Preprint 99-107

UFR-HEP/99/12

October 1999

\title{
Charged and Pseudoscalar Higgs production at a Muon Collider
}

\author{
A.G. Akeroyd ${ }^{\mathrm{a}}$, A. Arhrib ${ }^{\mathrm{b}, \mathrm{c}}$ and C. Dove \\ a: KEK Theory Group, Tsukuba, \\ Ibaraki 305-0801, Japan \\ b: Département de Mathématiques, Faculté des Sciences et Techniques \\ B.P 416, Tanger, Morocco \\ c: UFR-High Energy Physics, Physics Departement, Faculty of Sciences \\ PO Box 1014, Rabat, Morocco
}

\begin{abstract}
We consider single charged Higgs $\left(H^{ \pm}\right)$and pseudoscalar Higgs $\left(A^{0}\right)$ production in association with a gauge boson at $\mu^{+} \mu^{-}$colliders. We find that the tree-level tchannel and s-channel contributions to $\mu^{+} \mu^{-} \rightarrow H^{ \pm} W^{\mp}, A^{0} Z$ are enhanced for large values of $\tan \beta$, allowing sizeable cross-sections whose analogies at $e^{+} e^{-}$colliders would be very small. These processes provide attractive new ways of producing such particles at $\mu^{+} \mu^{-}$colliders and are superior to the conventional methods in regions of parameter space.
\end{abstract}




\section{Introduction}

Charged Higgs bosons $\left(H^{ \pm}\right)$are predicted in many favourable extensions of the Standard Model (SM), in particular the Minimal Supersymmetric Standard Model (MSSM). Their phenomenology [1] has received much attention both at $e^{+} e^{-}$colliders [2] and at hadron colliders [3], [4]. It is well known that $e^{+} e^{-}$colliders offer a much cleaner environment in which to look than hadron colliders, although recently progress has been made in the possibilities of detecting $H^{ \pm}$for $M_{H^{ \pm}} \geq m_{t}$ at hadron colliders [5]. At $e^{+} e^{-}$colliders production proceeds via the mechanism $e^{+} e^{-} \rightarrow \gamma^{*}, Z^{*} \rightarrow H^{+} H^{-}$, with higher order corrections evaluated in [6], and detection is possible for $M_{H^{ \pm}}$up to approximately $\sqrt{s} / 2$. The combined null-searches from all four LEP collaborations derive the lower limit $M_{H^{ \pm}} \geq$ $77.3 \mathrm{GeV}(95 \%$ c.l) [7].

In recent years an increasing amount of work has been dedicated to the physics possibilities of $\mu^{+} \mu^{-}$colliders [8], 9]. Such colliders offer novel ways of producing Higgs bosons, such as an $s$-channel resonance in the case of neutral scalars. The existing studies do not highlight any difference between the charged Higgs phenomenology at a $\mu^{+} \mu^{-}$ collider and $e^{+} e^{-}$collider, and state that the main production mechanism would be via $\mu^{+} \mu^{-} \rightarrow \gamma^{*}, Z^{*} \rightarrow H^{+} H^{-}$. The rate for this process is identical at both colliders. In the MSSM $H^{ \pm}$becomes roughly degenerate in mass with $H^{0}$ and $A^{0}$ for masses greater than $200 \mathrm{GeV}$. It is this correlation among the masses of the Higgs bosons which disallows any large effects from a $s^{-}$-channel resonance (via $\mu^{+} \mu^{-} \rightarrow H^{0}, h^{0} \rightarrow H^{+} H^{-}$) in the pair production mode, and we explicitly confirm this. In order for the above to be maximised one would require $\sqrt{s} \approx M_{h, H} \geq 2 M_{H^{ \pm}}$, a condition which requires sizeable mass splittings among the Higgs bosons and is disallowed in the MSSM.

So far unconsidered is the process $\mu^{+} \mu^{-} \rightarrow H^{ \pm} W^{\mp}$ via s-channel and t-channel diagrams. Naïvely, this may offer greater possibilities of a large rate since the Yukawa coupling only appears at one vertex in contrast to both vertices in the pair production case. In addition, it offers the possibility of searching for $M_{H^{ \pm}}$up to $\sqrt{s}-M_{W}$ in contrast to pair production which only probes up to $M_{H^{ \pm}} \leq \sqrt{s} / 2$. The rate for $b \bar{b} \rightarrow H^{ \pm} W^{\mp}$ at hadron colliders was considered in Ref. [10] although is not expected to provide an observable signature above the background [11], at least at LHC energies. In contrast, $\mu^{+} \mu^{-} \rightarrow H^{ \pm} W^{\mp}$ might give a clean signature, since backgrounds are considerably less.

In an analogous way we also consider $\mu^{+} \mu^{-} \rightarrow A^{0} Z$. The phenomenology of $A^{0}$ is made tricky at $e^{+} e^{-}$colliders due to the absence of a tree-level vertex $Z Z A^{0}$ and so the standard Higgsstrahlung mechanism $\left(e^{+} e^{-} \rightarrow A^{0} Z\right)$ only proceeds via loops [12]. Moreover, over a wide region of parameter space in the MSSM $A^{0}$ has a suppressed rate in the channel $\mu^{+} \mu^{-} \rightarrow A^{0} h^{0}$, while $\mu^{+} \mu^{-} \rightarrow A^{0} H^{0}$ only probes up to $M_{A} \approx \sqrt{s} / 2$. Proposed search strategies at $\mu^{+} \mu^{-}$collider include the scanning technique and Bremsstrahlung tail method. Since both may provide a challenge for machine and detector design we consider the prospects of searching for $A^{0}$ via $\mu^{+} \mu^{-} \rightarrow A^{0} Z$.

Our work is organized as follows. In Section 2 we perform the full tree-level calculation of $\mu^{+} \mu^{-} \rightarrow H^{+} H^{-}, \mu^{+} \mu^{-} \rightarrow H^{ \pm} W^{\mp}$ and $\mu^{+} \mu^{-} \rightarrow A^{0} Z$. In Section 3 we present numerical values of the cross-sections and Section 4 contains our conclusions. 


\section{Calculation}

We now consider in turn the various production mechanisms. Our calculations are valid in both the MSSM and a general Two-Higgs-Doublet-Model (2HDM), the difference being that the MSSM Higgs sector is parametrized by just two parameters at tree-level (usually taken as $M_{A}$ and $\tan \beta$ ), while the $2 \mathrm{HDM}$ contains 7 free parameters. Thus in a general 2HDM all four Higgs boson masses may be taken as independent, as well as the two mixing angles $\alpha$ and $\beta$, and the Higgs potential parameter $\lambda_{5}$ (in the notation of Ref. [13]). In addition, the Higgs trilinear couplings differ from those in the MSSM. In this paper we shall present numerical results for the MSSM. Let us summarise the couplings needed for our study:

\section{Fermion-Fermion-Higgs couplings}

$$
\begin{aligned}
h^{0} \mu^{+} \mu^{-} & =-\frac{i g m_{\mu}}{2 M_{W}} \lambda_{h \mu^{+} \mu^{-}} & H^{0} \mu^{+} \mu^{-}=-\frac{i g m_{\mu}}{2 M_{W}} \lambda_{H \mu^{+} \mu^{-}} \\
A^{0} \mu^{+} \mu^{-}=-\frac{i g m_{\mu}}{2 M_{W}} \gamma_{5} \lambda_{A^{0} \mu^{+} \mu^{-}} & , & H^{-} \mu^{+} \nu_{\mu}=\frac{i g m_{\mu}}{\sqrt{2} M_{W}} \lambda_{H^{+} \mu^{+} \nu_{\mu}} \frac{1-\gamma_{5}}{2}
\end{aligned}
$$

In the MSSM these couplings are given by:

$$
\begin{aligned}
& \lambda_{h \mu^{+} \mu^{-}}=-\frac{\sin \alpha}{\cos \beta}, \lambda_{H \mu^{+} \mu^{-}}=\frac{\cos \alpha}{\cos \beta} \\
& \lambda_{A^{0} \mu^{+} \mu^{-}}=\tan \beta, \lambda_{H^{-} \mu^{+} \nu_{\mu}}=\tan \beta
\end{aligned}
$$

One can see from the above formula that the $\mathrm{CP}$-odd $A^{0}$ and the charged Higgs bosons coupling to the $\mu^{ \pm}$can be enhanced for large $\tan \beta$.

The momenta of the incoming $\mu^{+}$and $\mu^{-}$, outgoing gauge boson $V\left(W^{ \pm}\right.$or $Z$ ) and outgoing Higgs scalar $S\left(H^{ \pm}\right.$or $\left.A^{0}\right)$ are denoted by $p_{\mu^{+}}, p_{\mu^{-}}, p_{V}$ and $p_{S}$, respectively. Neglecting the muon mass $m_{\mu}$, the momenta in the centre of mass of the $\mu^{+} \mu^{-}$system are given by:

$$
\begin{aligned}
& p_{\mu^{-}, \mu^{+}}=\frac{\sqrt{s}}{2}(1,0,0, \pm 1) \\
& p_{V, A^{0}}=\frac{\sqrt{s}}{2}\left(1 \pm \frac{M_{V}^{2}-M_{S}^{2}}{s}, \pm \frac{1}{s} \lambda^{\frac{1}{2}}\left(s, M_{V}^{2}, M_{S}^{2}\right) \sin \theta, 0, \pm \frac{1}{s} \lambda^{\frac{1}{2}}\left(s, M_{V}^{2}, M_{S}^{2}\right) \cos \theta\right),
\end{aligned}
$$

Here $\lambda(x, y, z)=x^{2}+y^{2}+z^{2}-2 x y-2 x z-2 y z$ is the two body phase space function and $\theta$ is the scattering angle between $\mu^{+}$and $S ; M_{V}$ is the mass of the gauge boson $V$ and $M_{S}$ is the mass of the Higgs scalar $S$. In the case of $H^{+} H^{-}$production replace $V$ by $S$. The Mandelstam variables are defined as follows:

$$
\begin{aligned}
& s=\left(p_{\mu^{-}}+p_{\mu^{+}}\right)^{2}=\left(p_{V}+p_{S}\right)^{2} \\
& t=\left(p_{\mu^{-}}-p_{V}\right)^{2}=\left(p_{\mu^{+}}-p_{S}\right)^{2}=\frac{1}{2}\left(M_{V}^{2}+M_{S}^{2}\right)-\frac{s}{2}+\frac{1}{2} \lambda^{\frac{1}{2}}\left(s, M_{V}^{2}, M_{S}^{2}\right) \cos \theta \\
& u=\left(p_{\mu^{-}}-p_{S}\right)^{2}=\left(p_{\mu^{+}}-p_{V}\right)^{2}=\frac{1}{2}\left(M_{V}^{2}+M_{S}^{2}\right)-\frac{s}{2}-\frac{1}{2} \lambda^{\frac{1}{2}}\left(s, M_{V}^{2}, M_{S}^{2}\right) \cos \theta \\
& s+t+u=M_{V}^{2}+M_{S}^{2}
\end{aligned}
$$




\section{$2.1 \quad \mu^{+} \mu^{-} \rightarrow H^{+} H^{-}$}

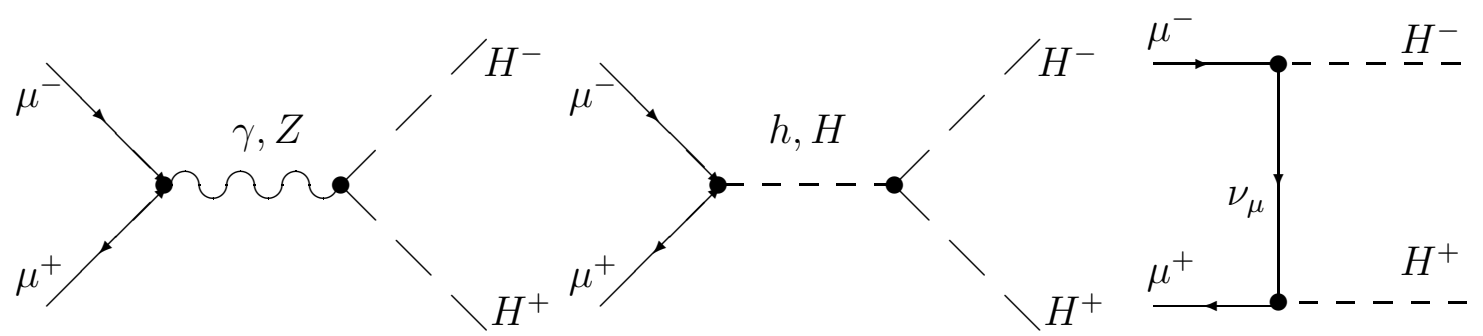

Figure.1

This process proceeds via the conventional Drell-Yan mechanism $\mu^{+} \mu^{-} \rightarrow \gamma^{*}, Z^{*} \rightarrow$ $H^{+} H^{-}$, the analogy of $e^{+} e^{-} \rightarrow \gamma^{*}, Z^{*} \rightarrow H^{+} H^{-}$. Since $m_{\mu} \approx 200 m_{e}$ one may consider the s-channel and t-channel diagrams (see Fig. 1), whose analogies at $e^{+} e^{-}$colliders would be suppressed by factors of $m_{e}$. The s-channel diagrams would be maximised for $\sqrt{s}=M_{h}$ or $M_{H}$, although in the context of the MSSM this condition would not allow on-shell pair production of $H^{ \pm}$. This can seen from the fact that $\sqrt{s} \geq 2 M_{H^{ \pm}}$and $\sqrt{s} \approx M_{h}$ or $M_{H}$ cannot be simultaneously satisfied in the MSSM. In contrast, such s-channel diagrams were considered in Ref. [14] for squark production via the process $\mu^{+} \mu^{-} \rightarrow \widetilde{q} \widetilde{q}$, and were shown to cause a doubling of the cross-section at resonance. The t-channel diagram in Fig. 1 suffers from Yukawa coupling suppression at two vertices. In the calculation we shall use the following notation:

$$
\begin{aligned}
& Y_{V}=-Y_{A}=\frac{m_{\mu}^{2}}{4 s_{W}^{2} M_{W}^{2}} \lambda_{H^{-} \mu \nu_{\mu}}^{2} \\
& a_{h}=-\frac{g_{h H^{+} H^{-}} m_{\mu} \lambda_{h \mu^{+} \mu^{-}}}{2 M_{W} s_{W}} \quad, \quad a_{H}=-\frac{g_{H H^{+} H^{-}} m_{\mu} \lambda_{H \mu^{+} \mu^{-}}}{2 M_{W} s_{W}} \\
& a_{1}=-2 \frac{1}{s}-\frac{1}{2 s_{W}^{2} c_{W}^{2}} \frac{g_{H} g_{V}}{s-M_{Z}^{2}+i M_{Z} \Gamma_{Z}}-\frac{Y_{V}}{t} \\
& a_{2}=\frac{1}{2 s_{W}^{2} c_{W}^{2}} \frac{g_{H} g_{A}}{s-M_{Z}^{2}+i M_{Z} \Gamma_{Z}}-\frac{Y_{A}}{t} \\
& a_{3}=\frac{a_{h}}{s-M_{h}^{2}+i M_{h} \Gamma_{h}}+\frac{a_{H}}{s-M_{H}^{2}+i M_{H} \Gamma_{H}}+\frac{m_{\mu} Y_{V}}{t}
\end{aligned}
$$

where $g_{V}=-\left(1-4 s_{W}^{2}\right) / 2, g_{A}=-1 / 2$ and $g_{H}=-c_{W}^{2}+s_{W}^{2}$. The coupling $g_{h H^{+} H^{-}}$and $g_{H^{+} H^{-}}$(normalised to electric charge e) are given by:

$$
\begin{aligned}
& g_{H H^{+} H^{-}}=-\frac{1}{s_{W}}\left\{M_{W} \cos (\beta-\alpha)-\frac{M_{Z}}{2 c_{W}} \cos 2 \beta \cos (\beta+\alpha)+\epsilon \frac{\cos \alpha \cos ^{2} \beta}{2 c_{W} M_{Z} \sin \beta}\right\} \\
& g_{h H^{+} H^{-}}=-\frac{1}{s_{W}}\left\{M_{W} \sin (\beta-\alpha)+\frac{M_{Z}}{2 c_{W}} \cos 2 \beta \sin (\beta+\alpha)+\epsilon \frac{\sin \alpha \cos ^{2} \beta}{2 c_{W} M_{Z} \sin \beta}\right\}
\end{aligned}
$$

Where

$$
\epsilon=\frac{3 G_{F} m_{t}^{4}}{\sqrt{2} \pi^{2} \sin ^{2} \beta} \log \left[\frac{m_{\tilde{t}_{1}} m_{\tilde{t}_{2}}}{m_{t}^{2}}\right]
$$


The $\epsilon$ term corresponds to the leading log 1-loop corrections 15 to the trilinear couplings. We will include also these leading log corrections to the Higgs-masses and to the mixing angles.

The square amplitude is given by:

$$
\begin{aligned}
|M|^{2}= & e^{4}\left\{\left(\left|a_{1}\right|^{2}+\left|a_{2}\right|^{2}\right) \frac{s^{2}}{2} \beta_{H}^{2} \sin ^{2} \theta-2\left|a_{2}\right|^{2} m_{\mu}^{2} s \beta_{H}^{2}+2\left|a_{3}\right|^{2} s\right. \\
& \left.+4 \Re\left(a_{1} a_{3}\right) m_{\mu} s \beta_{H} \cos \theta\right\}
\end{aligned}
$$

with $\beta_{H}^{2}=1-4 M_{H^{ \pm}}^{2} / s$. The differential cross-section is given by:

$$
\frac{d \sigma}{d \Omega}=\frac{\beta_{H}}{64 \pi^{2} s} \frac{1}{4}|M|^{2}
$$

\section{$2.2 \quad \mu^{+} \mu^{-} \rightarrow H^{ \pm} W^{\mp}$}
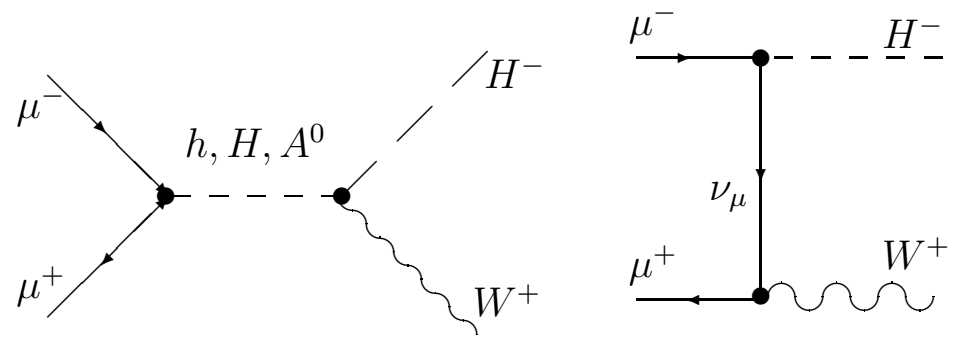

Figure.2

Single $H^{ \pm}$production may proceed via an s-channel resonance mediated by $h^{0}, H^{0}$ or $A^{0}$, and by t-channel exchange of $\nu_{\mu}$ (see Fig. 2). All are negligible at an $e^{+} e^{-}$collider due to the smallness of $m_{e}$. The loop induced contributions to $e^{+} e^{-} \rightarrow H^{ \pm} W^{\mp}$ were considered in Ref. [16] and shown to reach a few $\mathrm{fb}$ at very low values of $\tan \beta$, a region disfavoured in the MSSM. Potential advantages of $\mu^{+} \mu^{-} \rightarrow H^{ \pm} W^{\mp}$ over standard pair production are the following:

- $\mu^{+} \mu^{-} \rightarrow H^{ \pm} W^{\mp}$ is sensitive to the $H^{ \pm} \mu^{\mp} \nu_{\mu}$ Yukawa coupling, which is model dependent, and hence provides information on the underlying Higgs structure. For example, we shall see that a $2 \mathrm{HDM}$ with the Model I type structure would not register a signal in this channel. In contrast $\mu^{+} \mu^{-} \rightarrow \gamma^{*}, Z^{*} \rightarrow H^{+} H^{-}$has a model independent rate.

- Single $H^{ \pm}$production is less phase space suppressed than $H^{ \pm}$pair production, and would also allow greater kinematical reach at a given collider (on-shell production up to $\left.\sim \sqrt{s}-M_{W}\right)$.

- The $\mathrm{t}$-channel contribution may be sizeable and does not require $\sqrt{s} \approx M_{\text {res }}$ to be significant, where $M_{\text {res }}$ is the mass of a neutral Higgs s-channel resonance. This is in contrast to other novel production processes at $\mu^{+} \mu^{-}$colliders, which usually require the condition $\sqrt{s} \approx M_{\text {res }}$. 
The differential cross-section for $\sigma\left(\mu^{+} \mu^{-} \rightarrow H^{ \pm} W^{\mp}\right)$ may be written as follows:

$$
\frac{d \sigma}{d \Omega}=\frac{\lambda^{\frac{1}{2}}\left(s, M_{H^{ \pm}}^{2}, M_{W}^{2}\right)}{64 \pi^{2} s^{2}}|\mathcal{M}|^{2}
$$

The matrix element squared is given by:

$$
\begin{gathered}
|\mathcal{M}|^{2}=\frac{s g^{4} m_{\mu}^{2}}{32 M_{W}^{4}}\left[\left(\left|a_{V}\right|^{2}+\left|a_{A}\right|^{2}\right) \lambda\left(s, M_{H^{ \pm}}^{2}, M_{W}^{2}\right)+2 a_{t}^{2}\left(2 M_{W}^{2} p_{T}^{2}+t^{2}\right)\right. \\
\left.+2 a_{t}\left(M_{H^{ \pm}}^{2} M_{W}^{2}-s p_{T}^{2}-t^{2}\right) \Re\left(a_{V}-a_{A}\right)\right]
\end{gathered}
$$

Where $p_{T}^{2}=\lambda\left(s, M_{H^{ \pm}}^{2}, M_{W}^{2}\right) \sin ^{2} \theta / 4 s$ and the couplings $a_{V}, a_{A}$ and $a_{t}$ are given by:

$$
\begin{aligned}
a_{V} & =\left(\frac{\cos (\alpha-\beta) \lambda_{h \mu^{+} \mu^{-}}}{s-M_{h}^{2}+i M_{h} \Gamma_{h}}+\frac{\sin (\alpha-\beta) \lambda_{H \mu^{+} \mu^{-}}}{s-M_{H}^{2}+i M_{H} \Gamma_{H}}\right) \\
a_{A} & =\frac{\lambda_{A \mu^{+} \mu^{-}}}{s-M_{A}^{2}+i M_{A} \Gamma_{A}} \\
a_{t} & =\frac{\lambda_{H^{-} \mu^{+} \nu_{\mu}}}{t}
\end{aligned}
$$

The mixing angle dependence of the Higgs-Fermion-Fermion couplings is contained in $\lambda_{h \mu^{+} \mu^{-}}, \lambda_{H \mu^{+} \mu^{-}}, \lambda_{A \mu^{+} \mu^{-}}$and $\lambda_{H^{-} \mu^{+} \nu_{\mu}}$.

Our formula agrees with that for $b \bar{b} \rightarrow H^{ \pm} W^{\mp}$ in Ref. [10, with the replacements $m_{t} \rightarrow m_{\nu_{\mu}}$ and $m_{b} \rightarrow m_{\mu}$. Due to $\mathrm{CP}$-invariance the rate for $W^{+} H^{-}$and $W^{-} H^{+}$ production is identical. The total cross section takes the following form:

$$
\begin{aligned}
\sigma\left(\mu^{+} \mu^{-}\right. & \left.\rightarrow W^{+} H^{-}\right)=\frac{G_{F} m_{\mu}^{2}}{16 \pi s^{2}}\left\{\left(\left|a_{V}\right|^{2}+\left|a_{A}\right|^{2}\right) \lambda\left(s, M_{H^{ \pm}}^{2}, M_{W}^{2}\right) s\right. \\
& +2 \tan \beta\left[\Re\left(a_{A}-a_{V}\right)\left(M_{H^{ \pm}}^{2}+M_{W}^{2}-s\right) s+\left(s-4 M_{W}^{2}\right) \tan \beta\right] \lambda^{\frac{1}{2}}\left(s, M_{H^{ \pm}}^{2}, M_{W}^{2}\right) \\
& \left.-4 M_{W}^{2} \tan \beta\left[\Re\left(a_{V}-a_{A}\right) M_{H^{ \pm}}^{2} s+\left(M_{H^{ \pm}}^{2}+M_{W}^{2}-s\right) \tan \beta\right] F\left(s, M_{H^{ \pm}}^{2}, M_{W}^{2}\right)\right\}
\end{aligned}
$$

with:

$$
F\left(s, M_{S}^{2}, M_{V}^{2}\right)=\log \left[\frac{M_{S}^{2}+M_{V}^{2}-s-\lambda^{\frac{1}{2}}\left(s, M_{S}^{2}, M_{V}^{2}\right)}{M_{S}^{2}+M_{V}^{2}-s+\lambda^{\frac{1}{2}}\left(s, M_{S}^{2}, M_{V}^{2}\right)}\right]
$$

\section{$2.3 \mu^{+} \mu^{-} \rightarrow A^{0} Z$}

As depicted in Fig. 3, this process proceeds in a very similar way to that for $\mu^{+} \mu^{-} \rightarrow$ $H^{ \pm} W^{\mp}$, except there are two t-channel diagrams. The process $\mu^{+} \mu^{-} \rightarrow Z \phi^{0}$, where $\phi^{0}$ is the SM Higgs boson, has been considered in Ref. [17]. Our calculation differs since there is no s-channel $Z$ exchange for $\mu^{+} \mu^{-} \rightarrow A^{0} Z$ in the MSSM. Instead there are two s-channel Higgs exchange diagrams of similar magnitude to the $t$-channel diagram, giving rise to strong interference. In addition $\tan \beta$ plays an important role. In the SM the $\mathrm{s}-$ channel $Z$ exchange is the dominant diagram at the collider energy we consider $(\sqrt{s}=500 \mathrm{GeV})$, and so interference is minimal. 

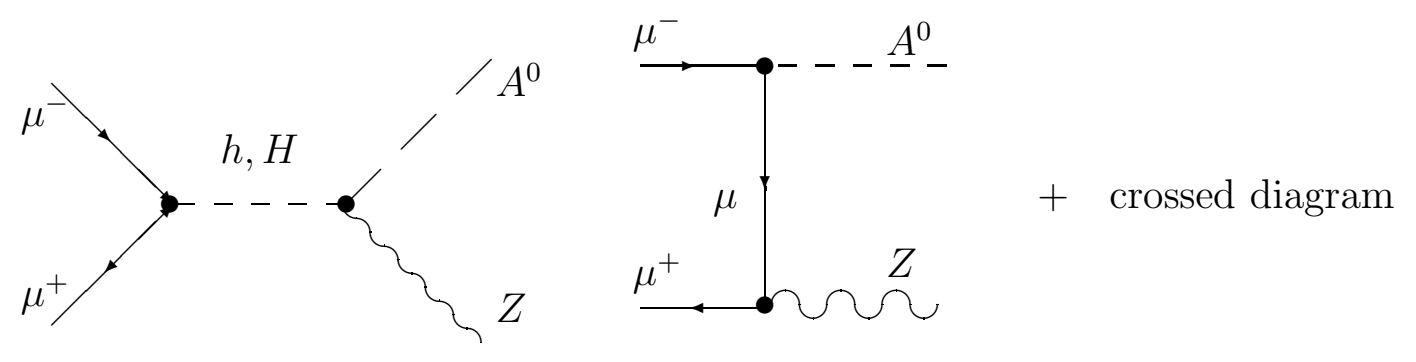

Figure.3

The mechanism $\mu^{+} \mu^{-} \rightarrow A^{0} Z$ would provide an alternative way of searching for $A^{0}$ whose detection is not guaranteed at the $\mathrm{LHC}$ or a $\sqrt{s}=500 \mathrm{GeV} e^{+} e^{-}$collider. At the latter this is because the conventional production mechanism $e^{+} e^{-} \rightarrow Z^{*} \rightarrow A^{0} H^{0}$ would be closed kinematically for $M_{A} \approx M_{H} \geq 250 \mathrm{GeV}$, and $e^{+} e^{-} \rightarrow Z^{*} \rightarrow A^{0} h^{0}$ $\left(\sim \cos ^{2}(\beta-\alpha)\right)$ is strongly suppressed for $M_{A} \geq 200 \mathrm{GeV}$. The proposed search at a $\mu^{+} \mu^{-}$collider for $M_{A} \geq \sqrt{s} / 2$ is by doing a scan over $\sqrt{s}$ energies, in order to find a resonance at $\sqrt{s}=M_{A}$, or by running the collider at full $\sqrt{s}$ and looking for peaks in the $b \bar{b}$ mass distribution (Bremsstrahlung tail method). These methods are competitive and both may allow detection up to $M_{A} \approx \sqrt{s}$ as long as $\tan \beta \geq 4-6$. However, both may provide quite a demanding challenge for detector resolution and machine design (see Ref. [8]), and it is too early to say with certainty if they would be feasible methods in practice. With this is mind we consider the process $\mu^{+} \mu^{-} \rightarrow A^{0} Z$. With a sizeable rate for $\sigma\left(\mu^{+} \mu^{-} \rightarrow A Z\right), A^{0}$ could be discovered first in this channel, and then the beams could be adjusted to $\sqrt{s}=M_{A}$ for precision studies. In addition, $\mu^{+} \mu^{-} \rightarrow A^{0} Z$ probes greater masses of $M_{A}$ than $e^{+} e^{-} \rightarrow Z^{*} \rightarrow A^{0} H^{0}$, and becomes another option to first discover $A^{0}$ (if discovery has been elusive at the LHC or a $\sqrt{s}=500 \mathrm{GeV} e^{+} e^{-}$collider). The matrix element squared may be written as:

$$
\begin{aligned}
|\mathcal{M}|^{2}= & \frac{s g^{4} m_{\mu}^{2}}{32 M_{W}^{4}}\left[\left|a_{V}\right|^{2} \lambda\left(s, M_{A}^{2}, M_{Z}^{2}\right)-2 a_{t 1} g_{A}\left(M_{A}^{2} M_{Z}^{2}-s p_{T}^{2}-t^{2}\right) \Re\left(a_{V}\right)\right. \\
& -2 a_{t 2} g_{A}\left(M_{A}^{2} M_{Z}^{2}-s p_{T}^{2}-u^{2}\right) \Re\left(a_{V}\right) \\
& +\left(g_{A}^{2}+g_{V}^{2}\right)\left\{a_{t 1}^{2}\left(2 M_{Z}^{2} p_{T}^{2}+t^{2}\right)+a_{t 2}^{2}\left(2 M_{Z}^{2} p_{T}^{2}+u^{2}\right)\right\} \\
& \left.-2\left(g_{A}^{2}-g_{V}^{2}\right) a_{t 1} a_{t 2}\left(2 M_{Z}^{2} p_{T}^{2}+2 M_{A}^{2} M_{Z}^{2}-t u\right)\right]
\end{aligned}
$$

with $a_{V}$ the same as in Section 2.2 and

$$
a_{t 1}=\frac{\lambda_{A \mu^{+} \mu^{-}}}{t-m_{\mu}^{2}} \quad, \quad a_{t 2}=\frac{\lambda_{A \mu^{+} \mu^{-}}}{u-m_{\mu}^{2}}
$$

The differential cross-section follows from eq(可) with the changes $M_{H^{ \pm}} \rightarrow M_{A}$ and $M_{W} \rightarrow$ $M_{Z}$. 
The total cross-section is given by:

$$
\begin{aligned}
\sigma\left(\mu^{+} \mu^{-} \rightarrow A^{0} Z\right) & =\frac{G_{F} m_{\mu}^{2}}{32 \pi s^{2}}\left\{\left[4 s\left(g_{A}^{2}-g_{V}^{2}\right) \tan ^{2} \beta+2 s\left|a_{V}\right|^{2} \lambda\left(s, M_{A}^{2}, M_{Z}^{2}\right)\right] \lambda^{\frac{1}{2}}\left(s, M_{A}^{2}, M_{Z}^{2}\right)\right. \\
& +\left[8 s \Re\left(a_{V}\right) g_{A}\left(M_{A}^{2}+M_{Z}^{2}-s\right) \tan \beta-8\left(g_{A}^{2}-g_{V}^{2}\right) M_{Z}^{2} \tan ^{2} \beta\right. \\
& \left.+4\left(g_{A}^{2}+g_{V}^{2}\right)\left(s-4 M_{Z}^{2}\right) \tan ^{2} \beta\right] \lambda^{\frac{1}{2}}\left(s, M_{A}^{2}, M_{Z}^{2}\right) \\
& +\frac{F\left(s, M_{A}^{2}, M_{Z}^{2}\right)}{\left(M_{A}^{2}+M_{Z}^{2}-s\right)}\left[-8 M_{Z}^{2} \tan \beta\left(-2 \Re\left(a_{V}\right) g_{A} M_{A}^{2}\left(M_{A}^{2}+M_{Z}^{2}-s\right) s\right.\right. \\
& \left.\left.\left.+\left(2\left(g_{A}^{2}-g_{V}^{2}\right) M_{A}^{2}\left(M_{Z}^{2}-s\right)+\left(g_{A}^{2}+g_{V}^{2}\right)\left(M_{A}^{2}+M_{Z}^{2}-s\right)^{2}\right) \tan \beta\right)\right]\right\}
\end{aligned}
$$

\section{$3 \quad$ Numerical results}

We now present our numerical analysis in the context of the MSSM. We take $\sqrt{s}=500$ $\mathrm{GeV}$ and assume integrated luminosities of the order $50 \mathrm{fb}^{-1}$.

In Fig. 4 we plot $\sigma\left(\mu^{+} \mu^{-} \rightarrow H^{ \pm} W^{\mp}\right)$, defined as the sum of $H^{+} W^{-}$and $H^{-} W^{+}$ production, as a function of $M_{H^{ \pm}}$, varying $\tan \beta$ from 20 to 50 . We also include the tree-level rate for $\sigma\left(e^{+} e^{-} \rightarrow H^{+} H^{-}\right)$in order to show the advantage of a $\mu^{+} \mu^{-}$collider over an $e^{+} e^{-}$collider. One can see that the single production mode gains in importance with increasing $\tan \beta$, and offers detection possibilities for $M_{H^{ \pm}}$up to $\sqrt{s}-M_{W}$. This compares favourably with the reach at an $e^{+} e^{-}$collider.

The slight dip and rise of the curves arises due to the $H^{0}$ and $A^{0}$ mediated s-channel contributions increasing in magnitude with $M_{H^{ \pm}}$, which compensates for the phase space suppression until the kinematical limit is approached. This can be seen from the fact that since $M_{H^{ \pm}} \approx M_{H} \approx M_{A}$, larger $M_{H^{ \pm}}$causes both $M_{H}$ and $M_{A}$ to be closer to $\sqrt{s}$ (i.e. the resonance condition).

It is clear from the graphs that for $\tan \beta \geq 20$ one has $\sigma\left(\mu^{+} \mu^{-} \rightarrow H^{ \pm} W^{\mp}\right) \geq 5$ $\mathrm{fb}$, which would give a sizeable number of singly produced $H^{ \pm}$for luminosities of 50 $\mathrm{fb}^{-1}$. One would expect $H^{ \pm} \rightarrow t b$ decays for the mass region of interest and so the main background would be from $t \bar{t}$ production. Such a background 11] was shown to overwhelm the channel $p p \rightarrow H^{ \pm} W^{\mp}$ 10 at the LHC. However, at a $\sqrt{s}=500 \mathrm{GeV}$ muon collider $\sigma\left(\mu^{+} \mu^{-} \rightarrow t \bar{t}\right) \sim 0.7 \mathrm{pb}$ in contrast to $\sim 800 \mathrm{pb}$ at the LHC. Hence we would expect much better prospects for detection at a muon collider although a full signal-background analysis is beyond the scope of this paper. Previous studies of backgrounds to $H^{ \pm} W^{\mp}$ production at $e^{+} e^{-}$colliders have been carried out in the context of Higgs triplet models [18], assuming $H^{ \pm} \rightarrow W^{ \pm} Z$ as the main decay channel. Such studies cannot be applied to the MSSM where $H^{ \pm} \rightarrow t b$ decays would dominate.

We note that a $2 \mathrm{HDM}$ with the Model I type structure would not register an observable signal in this channel. This is due to the rate being proportional to $\cot ^{2} \beta$, and so unacceptably small values of $\tan \beta$ would be required in order to allow observable cross-sections.

The process $\mu^{+} \mu^{-} \rightarrow A^{0} Z$ suffers from smaller cross-sections and these are plotted as a function of $M_{A}$ in Fig. 5. Given that $\mu^{+} \mu^{-} \rightarrow A^{0} H^{0}$ probes $M_{A}$ up to $\approx \sqrt{s} / 2$ the 


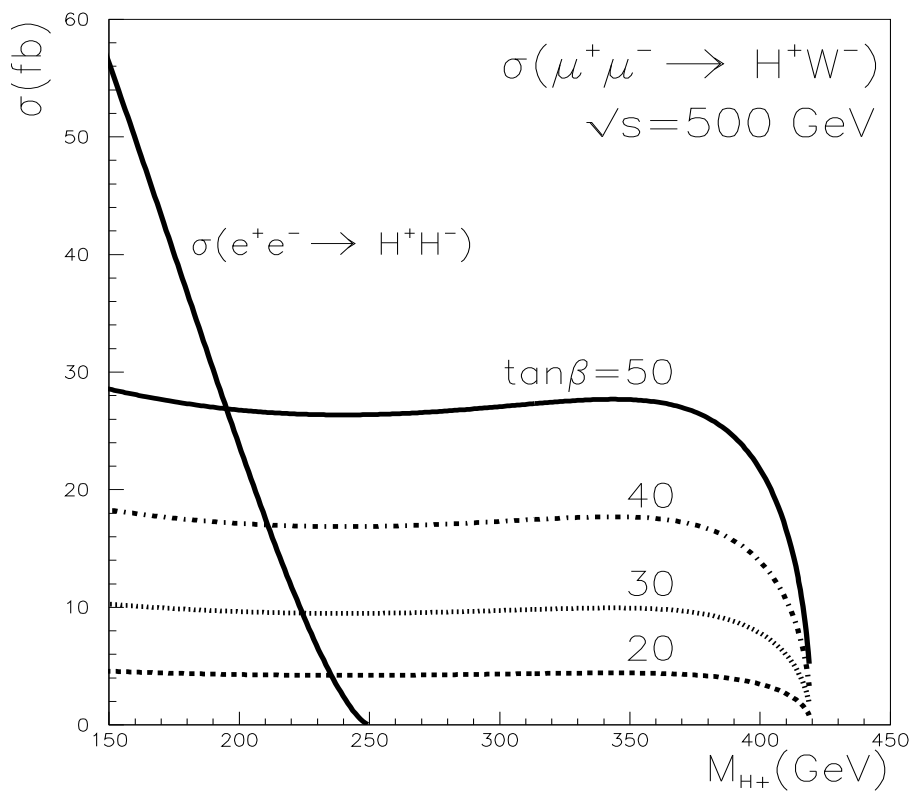

Figure 4: $\sigma\left(\mu^{+} \mu^{-} \rightarrow H^{ \pm} W^{\mp}\right)$ as a function of $M_{H^{ \pm}}$for various values of $\tan \beta$. Also indicated is $\sigma\left(e^{+} e^{-} \rightarrow H^{+} H^{-}\right)$for the same $\sqrt{s}$.

region $M_{A} \geq 250 \mathrm{GeV}$ is of interest. We see that cross-sections $\geq 1 \mathrm{fb}$ are only attainable in this region for $\tan \beta \geq 30$ and so detection would be restricted to large values of $\tan \beta$. The smallness of the cross-sections is caused by large destructive interference between the $s$ and $t$ channels.

Finally, we consider $\mu^{+} \mu^{-} \rightarrow H^{+} H^{-}$. We find very small deviations from the rate for $e^{+} e^{-} \rightarrow H^{+} H^{-}$, of the order a few percent for large values of $\tan \beta$. This can be traced to the fact that the $\mathrm{s}$-channel Higgs exchange diagrams are far from resonance, and the t-channel diagrams are doubly Yukawa suppressed. Since the 1-loop corrections [6] may be much larger than these deviations we do not plot a graph.

\section{Conclusions}

We have considered the processes $\mu^{+} \mu^{-} \rightarrow H^{ \pm} W^{\mp}$ and $\mu^{+} \mu^{-} \rightarrow A^{0} Z$ of the MSSM in the context of a high-energy $\mu^{+} \mu^{-}$collider $(\sqrt{s}=500 \mathrm{GeV})$. We showed that $\mu^{+} \mu^{-} \rightarrow H^{ \pm} W^{\mp}$ production offers an attractive new way of searching for $H^{ \pm}$at such colliders. The crosssection grows with increasing $\tan \beta$ with values as large as $30 \mathrm{fb}$ being attainable for $\tan \beta \geq 50$. With an integrated luminosity of $50 \mathrm{fb}^{-1}$ a significant number of $H^{ \pm}$could be produced singly up to $M_{H^{ \pm}} \approx \sqrt{s}-M_{W}$. This compares favourably with the reach 


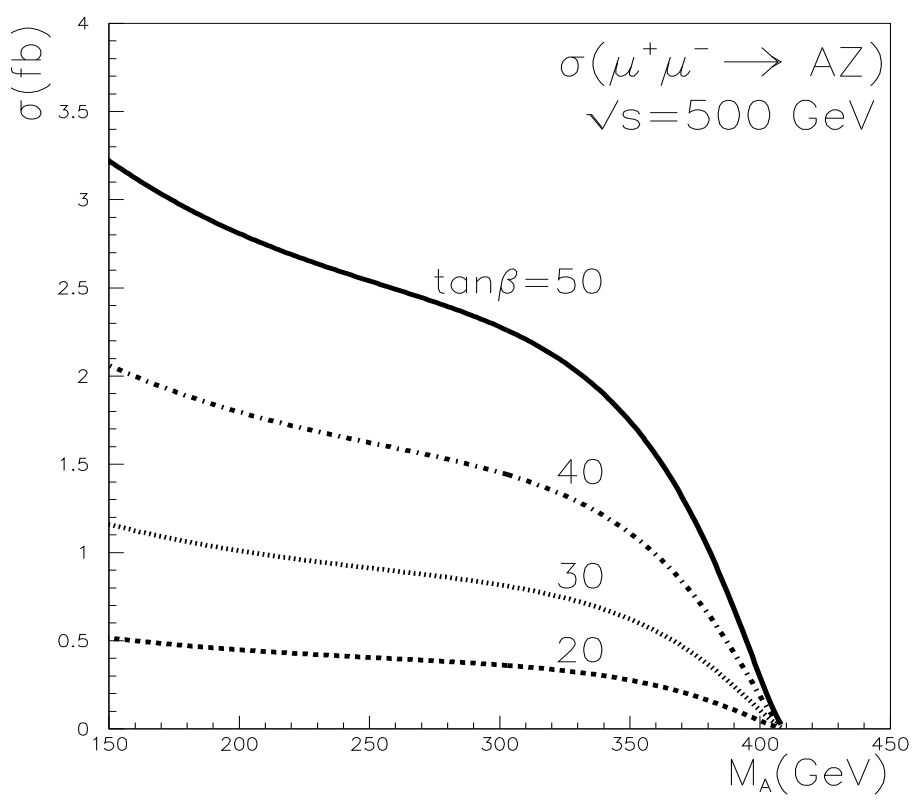

Figure 5: $\sigma\left(\mu^{+} \mu^{-} \rightarrow A^{0} Z\right)$ as a function of $M_{A}$ for various values of $\tan \beta$.

at an $e^{+} e^{-}$collider, which may only probe up to $M_{H^{ \pm}} \approx \sqrt{s} / 2$. The main background (assuming $H^{ \pm} \rightarrow t b$ decays) would be from $t \bar{t}$ production, which has a cross-section of $700 \mathrm{fb}, 3$ orders of magnitude less than at the LHC. We conclude that the mechanism $\mu^{+} \mu^{-} \rightarrow H^{ \pm} W^{\mp}$ represents a novel and attractive way of producing $H^{ \pm}$at a $\mu^{+} \mu^{-}$ collider, and in our opinion merits a detailed signal-background analysis.

Pseudoscalar Higgs production via $\mu^{+} \mu^{-} \rightarrow A^{0} Z$ offers smaller cross-sections, with values of $2 \mathrm{fb}$ or more only possible for large $(\geq 40) \tan \beta$. Charged Higgs pair production has essentially the same rate as that at an $e^{+} e^{-}$collider, with differences of the order of a few percent for large values of $\tan \beta$.

\section{Acknowledgements}

A.G. Akeroyd was supported by the Japan Society for Promotion of Science (JSPS). We thank A. Turcot for useful comments.

\section{References}

[1] J.F. Gunion, H.E. Haber, G.L. Kane and S. Dawson, The Higgs Hunter's Guide (Addison-Wesley, Reading, 1990). 
[2] S. Komamiya, Phys. Rev. D38 (1988) 2158; A. Sopczak, Z.Phys. C65 (1995) 449; S. Moretti and K. Odagiri, J. Phys. G23 (1997) 537.

[3] E. Eichten, I. Hinchliffe, K. Lane and C. Quigg, Rev. Mod. Phys. 56 (1984) 579; J. Gunion, H.E. Haber, F.E. Paige, W.K. Tung and S.S.D. Willenbrock, Nucl. Phys. B294 (1987) 621; R.M. Barnett, H.E. Haber and D.E. Soper, B306 (1988) 697; D.A. Dicus, J.L. Hewett, C. Kao, and T.G. Rizzo, Phys. Rev. D40 (1989) 787; V. Barger, R.J.N. Philips and D.P. Roy, Phys. lett. B324 (1994) 236; J.L. Diaz-Cruz and O.A. Sampayo, Phys. Rev. D50 (1994) 6828.

[4] Jiang Yi, Ma Wen-Gan, Han Liang, Han Meng and Yu Zeng-hui; J. Phys. G24 (1998) 83; J. Phys. G23 (1997)385; A. Krause, T. Plehn, M. Spira and P.W. Zerwas, Nucl. Phys. B519 (1998) 85; S. Moretti and K. Odagiri, Phys. Rev. D55 (1997) 5627; Li Gang Jin, Chong Sheng Li, R.J. Oakes and Shou Hua Zhu, hep-ph/9907482; A.A. Barrientos Bendezu and B.A. Kniehl, hep-ph/9908385; O. Brein and W. Hollik, hep-ph/9908529.

[5] K. Odagiri. Phys. Lett. B452 (1999) 327; K. Odagiri, hep-ph/9901432; D.P. Roy, Phys. Lett. B459 (1999) 607; D.J. Miller, S. Moretti, D.P. Roy and W.J. Stirling, hep-ph/9906230; M. Drees, M. Guchait and D.P. Roy, hep-ph/9909266, S. Moretti and D.P. Roy, hep-ph/9909435.

[6] A. Arhrib, M. Capdequi Peyranère and G. Moultaka, Phys. Lett. B341 (1995) 313; M.A. Diaz and Tonnis A. ter Veldhuis, hep-ph/9501315; A. Arhrib and G. Moultaka, hep-ph/9808317, to appear in Nucl. Phys. B.

[7] Combined Experimental Limits; ALEPH 99-081 CONF 99-052; DELPHI 99-142 CONF 327; L3 Note 2442; OPAL Technical Note TN-614.

[8] Proceedings of the Workshop on Physics at the First Muon Collider and front end of a Muon Collider, Fermilab, November 6-9, 1997; $\mu^{+} \mu^{-}$Collider: a Feasibility Study, BNL-52503, Fermilab-Conf-96/092, LBNL-38946, July 1996; Phys. Rep. 286 (1996) 1.

[9] J. Gunion, hep-ph/9802258, V. Barger, hep-ph/9803480.

[10] A.A. Barrientos Bendezu and B.A. Kniehl, Phys. Rev. D59 (1999) 015009.

[11] S. Moretti and K. Odagiri, Phys. Rev. D59 (1999) 055008.

[12] A.G. Akeroyd, A. Arhrib and M. Capdequi Peyranère, Mod. Phys. Lett. A14 (1999) 2093.

[13] A. Djouadi, V. Driesen, W. Hollik and A. Kraft, Eur. Phys. J. C1 (1998) 163.

[14] A. Bartl, H. Eberl, K. Hidaka, S. Kraml, W. Majerotto, W.Porod and Y. Yamada, Phys. Rev. D58 (1999) 115002. 
[15] Y. Okada, M. Yamaguchi and T. Yanagida, Prog. Theo. Phys. 85 (1991) 1; H. Haber and R. Hempling, Phys. Rev. Lett. 66 (1991) 1815; J. Ellis, G. Ridolfi and F. Zwirner, Phys. Lett. B257 (1991) 83; R. Barbieri, F. Caravaglios and M. Frigeni, Phys. Lett. B258 (1991) 167.

[16] Shou Hua Zhou, hep-ph/9901221.

[17] V.A. Litvin and F.F. Tikhonin, hep-ph/9704417; F.F. Tikhonin, IFVE-98-18; V.A. Litvin and F.F. Tikhonin, IFVE-98-19.

[18] K. Cheung, R.J.N. Phillips and A. Pilaftsis, Phys. Rev. D51 (1995) 4731; D.K. Ghosh, R.M. Godbole and B. Mukhopadhyaya, Phys. Rev. D55 (1997) 3150. 